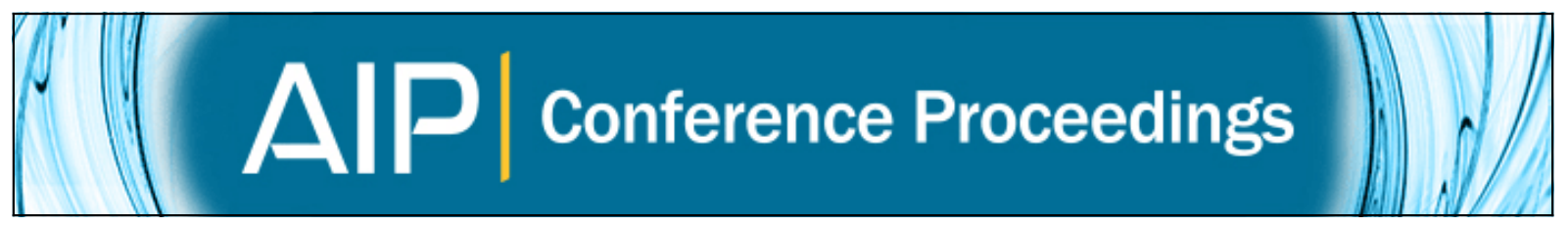

\title{
Evaporation/condensation boundary conditions for the regularized 13 moment equations
}

Henning Struchtrup and Aldo Frezzotti

Citation: AIP Conference Proceedings 1786, 140002 (2016); doi: 10.1063/1.4967633

View online: http://dx.doi.org/10.1063/1.4967633

View Table of Contents: http://scitation.aip.org/content/aip/proceeding/aipcp/1786?ver=pdfcov

Published by the AIP Publishing

\section{Articles you may be interested in}

Thermodynamically admissible boundary conditions for the regularized 13 moment equations

Phys. Fluids 28, 027105 (2016); 10.1063/1.4941293

Numerical analysis of kinetic boundary conditions at net evaporation/condensation interfaces in various liquid temperatures based on mean-field kinetic theory

AIP Conf. Proc. 1628, 398 (2014); 10.1063/1.4902620

Method of determining kinetic boundary conditions in net evaporation/condensation

Phys. Fluids 26, 072003 (2014); 10.1063/1.4890523

Analytical and Numerical Solutions of Boundary Value Problems for the Regularized 13 Moment Equations AIP Conf. Proc. 1333, 627 (2011); 10.1063/1.3562717

Grooving of a grain boundary by evaporation-condensation below the roughening transition J. Appl. Phys. 97, 113535 (2005); 10.1063/1.1922583 


\title{
Evaporation/Condensation Boundary Conditions for the Regularized 13 Moment Equations
}

\author{
Henning Struchtrup ${ }^{1, a)}$ and Aldo Frezzotti ${ }^{2, b)}$ \\ ${ }^{1}$ Dept. of Mechanical Engineering, University of Victoria, PO Box 1700, Stn. CSC, Victoria, BC, V8W 2Y2, Canada \\ ${ }^{2}$ Dipartimento di Scienze e Tecnologie Aerospaziali, Politecnico di Milano, Via La Masa 34, 20156 Milano, Italy \\ a) struchtr@uvic.ca \\ b)aldo.frezzotti@polimi.it
}

\begin{abstract}
The regularized 13 moment equations (R13) are a macroscopic model for the description of rarefied gas flows in the transition regime. The equations have been shown to give meaningful results for Knudsen numbers up to about 0.5 . Here, their range of applicability is extended by boundary conditions for evaporating and condensing interfaces, derived from the microscopic interface conditions of kinetic theory. Simple 1-D problems are used to test the R13 equations with evaporation and condensation.
\end{abstract}

\section{Introduction}

The regularized 13 moment (R13) equations are a macroscopic model to describe rarefied gas flows for not too large Knudsen numbers in good approximation to the Boltzmann equation [1, 2, 3, 4, 5]. Gas rarefaction leads to the occurrence of phenomena such as velocity slip and temperature jump at boundaries, Knudsen layers in front of boundaries, transpiration flow, thermal stresses, or heat transfer without temperature gradients, all of which are reproduced by solutions of the R13 equations, but cannot be accurately described by the Navier-Stokes-Fourier (NSF) equations of classical hydrodynamics [3]. Proper modelling of boundary conditions is essential to obtain a meaningful description of rarefied flows, and below we present and test conditions for liquid-vapor boundaries with condensation and evaporation. With this, the range of application of the R13 equations is extended in particular towards microdevices with phase change.

The R13 equations are derived as approximations of the Boltzmann by means of the order of magnitude method $[6,7]$, which combines elements of the Chapman-Enskog [8, 9, 10, 11, 12] and Grad [13] methods; the resulting equations avoid the problems exhibited by the individual methods.

Just as the transport equations are derived from the Boltzmann equation, the corresponding macroscopic boundary and interface conditions are derived from the microscopic boundary and interface conditions for the Boltzmann equation [14]. For this we use an extended Maxwell boundary model [15] with an condensation/evaporation coefficient and an accommodation coefficient [16]. The derivation follows the same line as that of the wall boundary conditions for non-condensing interfaces. Naturally, for vanishing condensation coefficient, the boundary conditions for solid walls are recovered.

We solve two simple one-dimensional evaporation problems to test the R13 equations with evaporation and condensation. Solutions of R13 and NSF are compared with DSMC simulations, and it is shown that generally R13 gives good agreement to the kinetic solutions, both for overall parameters like the mass and heat flows, and for details of the flows, such as temperature and stress profiles with Knudsen layers.

\section{The R13 equations}

The aim of kinetic theory is to find the velocity distribution function $f\left(x_{i}, t, c_{i}\right)$, where $t$ denotes time $t, x_{i}$ is the location in space and $c_{i}$ is the microscopic velocity. In a microscopic approach, the distribution function is the solution of the Boltzmann equation $[8,9]$, while in a macroscopic approach one derives transport equations as a set of suitable 
moments of the Boltzmann equation, where the resulting moment equations are closed by means of an ansatz for the distribution [13,3]. The basic 13 variables are mass density $\rho$, macroscopic velocity $v_{i}$, temperature $T$, anisotropic stress tensor $\sigma_{i j}$ (with $\sigma_{i i}=0$ ), and heat flux vector $q_{i}$, which are moments of the distribution function, as

$$
\rho=m \int f d \mathbf{c}, \rho v_{i}=m \int c_{i} f d \mathbf{c}, \rho u=\frac{m}{2} \int C^{2} f d \mathbf{c}, \sigma_{i j}=m \int C_{\langle i} C_{j\rangle} f d \mathbf{c}, q_{i}=\frac{m}{2} \int C^{2} C_{i} f d \mathbf{c}
$$

Here, $u=\frac{3}{2} R T=\frac{3}{2} \theta$ is the specific internal energy, $\theta=R T$ is temperature in energy units with the specific gas constant $R$, and $C_{i}=c_{i}-v_{i}$ is the peculiar velocity. Indices in angular brackets denote the symmetric, trace-free part of a tensor [3].

The corresponding moment equations are the conservation laws for mass, momentum and energy, which can be written as $\left(\frac{D}{D t}=\frac{\partial}{\partial t}+v_{k} \frac{\partial}{\partial x_{k}}\right.$ is the material time derivative)

$$
\begin{aligned}
\frac{D \rho}{D t}+\rho \frac{\partial v_{k}}{\partial x_{k}} & =0, \\
\rho \frac{D v_{i}}{D t}+\rho \frac{\partial \theta}{\partial x_{i}}+\theta \frac{\partial \rho}{\partial x_{i}}+\frac{\partial \sigma_{i k}}{\partial x_{k}} & =0, \\
\frac{3}{2} \rho \frac{D \theta}{D t}+\rho \theta \frac{\partial v_{k}}{\partial x_{k}}+\frac{\partial q_{k}}{\partial x_{k}}+\sigma_{k l} \frac{\partial v_{k}}{\partial x_{l}} & =0,
\end{aligned}
$$

and the balance equations for stress and heat flux

$$
\begin{gathered}
\frac{D \sigma_{i j}}{D t}+\sigma_{i j} \frac{\partial v_{k}}{\partial x_{k}}+2 \sigma_{k\langle i} \frac{\partial v_{j\rangle}}{\partial x_{k}}+\frac{4}{5} \frac{\partial q_{\langle i}}{\partial x_{j\rangle}}+\frac{\partial m_{i j k}}{\partial x_{k}}=-\rho \theta\left[\frac{\sigma_{i j}}{\mu}+2 \frac{\partial v_{\langle i}}{\partial x_{j\rangle}}\right], \\
\frac{D q_{i}}{D t}+\frac{5}{2} \sigma_{i k} \frac{\partial \theta}{\partial x_{k}}-\sigma_{i k} \theta \frac{\partial \ln \rho}{\partial x_{k}}+\theta \frac{\partial \sigma_{i k}}{\partial x_{k}}+\frac{14}{5} q_{\langle i} \frac{\partial v_{k\rangle}}{\partial x_{k}}+\frac{4}{3} q_{k} \frac{\partial v_{k}}{\partial x_{i}}+\frac{1}{2} \frac{\partial R_{i k}}{\partial x_{k}}+\frac{1}{6} \frac{\partial \Delta}{\partial x_{i}}+m_{i k l} \frac{\partial v_{k}}{\partial x_{l}}-\frac{\sigma_{i k}}{\rho} \frac{\partial \sigma_{k l}}{\partial x_{l}}=-\frac{5}{2} \rho \theta\left[\frac{q_{i}}{\kappa}+\frac{\partial \theta}{\partial x_{i}}\right]
\end{gathered}
$$

The collision terms of the above equations were determined for Maxwell molecules, $\mu$ is the shear viscosity, and $\kappa=\frac{15}{4} \mu$ is the heat conductivity [3].

For small Knudsen numbers, the Chapman-Enskog method [8,3] can be used to reduce the equations for stress and heat flux to the Navier-Stokes and Fourier laws,

$$
\sigma_{i j}=-2 \mu \frac{\partial v_{\langle i}}{\partial x_{j\rangle}}, \quad q_{i}=-\kappa \frac{\partial \theta}{\partial x_{i}} .
$$

In addition to the 13 variables introduced above, the 13 moment equations $(3,5,6)$ contain the additional moments

$$
m_{i j k}=m \int C_{\langle i} C_{j} C_{k\rangle} f d \mathbf{c}, \Delta=m \int C^{4} f d \mathbf{c}-15 \rho \theta^{2}, R_{i j}=m \int C^{2} C_{\langle i} C_{j\rangle} f d \mathbf{c}-7 \sigma_{i j}
$$

In order to close the system of moment equations, constitutive equations for these variables must be provided. The classical Grad closure [13,3] simply leads to $m_{i j k \mid G}=\Delta_{\mid G}=R_{i j \mid G}=0$. The regularized 13 moment equations arise from an alternative closure, which accounts for parts of, but not the complete, transport equations for $\left\{m_{i j k}, \Delta, R_{i j}\right\}[1,6,7$, 17]. The regularized 13 moment equations arise as the appropriate set of equations at 3rd order in the Knudsen number (super-Burnett order), and, for Maxwell molecules, consist of the equations $(3,5,6)$ and the constitutive equations (pressure obeys the ideal gas law, $p=\rho \theta$ )

$$
\begin{aligned}
\Delta & =5 \frac{\sigma_{k l} \sigma_{k l}}{\rho}+\frac{56}{5} \frac{q_{k} q_{k}}{p}-12 \mu \theta \frac{\partial}{\partial x_{k}}\left(\frac{q_{k}}{p}\right), \\
R_{i j} & =\frac{20}{7} \frac{\sigma_{k\langle i} \sigma_{j\rangle k}}{\rho}+\frac{64}{25} \frac{q_{\langle i} q_{j\rangle}}{p}-\frac{24}{5} \mu \theta \frac{\partial}{\partial x_{\langle i}}\left(\frac{q_{j\rangle}}{p}\right), \\
m_{i j k} & =\frac{4}{3} \frac{\sigma_{\langle i j} q_{k\rangle}}{p}-2 \mu \theta \frac{\partial}{\partial x_{\langle i}}\left(\frac{\sigma_{j k\rangle}}{p}\right) .
\end{aligned}
$$




\section{Microscopic and macroscopic boundary conditions}

At an evaporating liquid interface, some vapor particles that hit the interface condense, while those that do not condense are reflected; moreover, some particles are injected into the vapor by evaporation from the liquid. We write the distribution function directly in front of the interface as

$$
f_{\text {int }}= \begin{cases}f^{-}, & C_{n}^{I \prime} \leq 0 \\ f^{+}, & C_{n}^{I}>0\end{cases}
$$

where $f^{-}$is the distribution of incident particles (negative velocity $C_{n}^{I \prime}$ normal, and relative, to the interface), and $f^{+}$is the distribution of emitted particles (positive velocity $C_{n}^{I}$ normal to the interface). For finding boundary conditions for moments we shall describe the gas by the distribution function associated with the R13 equations, $f^{-}=f_{\mid R 13}$, which is a Grad distribution for 26 moments, which are the 13 variables plus the 13 constitutive quantities (10) [3].

The distribution of particles leaving the interface is the sum of evaporation and reflection of non-condensing particles back into the vapor. For the latter we follow the classical Maxwell model, which assumes that particles are either specularly reflected, or thermalized and leave in a Maxwellian $[15,16]$. The emitted distribution function thus can be written as

$$
f^{+}=\vartheta f_{M}\left(p_{\text {sat }}\left(\theta_{L}\right), \theta_{L}, C^{I}\right)+(1-\vartheta)(1-\chi) f_{\mid R 13}\left(C_{i}^{I}-2 C_{n}^{I} n_{i}\right)+(1-\vartheta) \chi f_{M}\left(\bar{p}, \theta_{L}, C^{I}\right),
$$

where $\vartheta$ is the evaporation/condensation probability, and $\chi$ is the accommodation coefficient, defined as the probability that a reflected particle is thermalized. The velocity $C_{i}^{I}$ is the velocity of a vapor particle as seen from an observer resting with the liquid-vapor interface; $C_{n}^{I}=C_{j}^{I} n_{j}$ is the velocity normal to the interface; $C_{i}^{I}-2 C_{n}^{I} n_{i}$ is the specular reflection velocity, the notation is such that $f_{\mid R 13}\left(C_{i}^{I}-2 C_{n}^{I} n_{i}\right)$ denotes the distribution of specularly reflected particles. The pressure $\bar{p}$ in the Maxwellian for thermalized particles is determined from the condition that non-condensing particles must return to the vapor.

The liquid side of the interface is assumed to be in local equilibrium [18, 19], and thus evaporating particles leave in a Maxwellian distribution $f_{M}\left(p_{\text {sat }}\left(\theta_{L}\right), \theta_{L}, C^{I}\right)$, where $p_{\text {sat }}\left(\theta_{L}\right)$ is the saturation pressure corresponding to liquid temperature $\theta_{L}$. For thermal equilibrium, the outgoing distribution reduces to $f_{\mid E}^{+}=f_{\mid R 13, E}=f_{M}\left(p_{\text {sat }}\left(\theta_{L}\right), \theta_{L}, C\right)$.

Continuity conditions for fluxes of moments are used to find the boundary conditions for the moments [13, 14 , $4,5]$. For an observer slipping with the gas along the liquid-vapor interface, who observes the particle velocity $\hat{C}_{i}$, the normal flux computed with the distribution function directly at the wall, i.e., the distribution $f_{\text {int }}$ of Eq. (12), must be equal to the normal flux computed with the distribution function $f_{\mid R 13}$ of the gas just in front of the wall [3], which leads to

$$
\int_{\hat{C}_{n}>0} \hat{\Psi}_{A} \hat{C}_{n} f^{+} d \mathbf{c}=\int_{\hat{C}_{n}>0} \hat{\Psi}_{A} \hat{C}_{n} f_{\mid R 13} d \mathbf{c} .
$$

Here, following Grad [13], we have to use continuity only of fluxes that are odd in $\hat{C}_{n}$, which implies functions $\hat{\Psi}_{A}$ which are even in $\hat{C}_{n}$. The 13 variables of the R13 equations are moments based on the weights $\phi_{A}=$ $m\left\{1, c_{i}, c^{2}, c_{i} c_{j}, c^{2} c_{i}\right\}_{A}$, and of corresponding the even tensor components are $\phi_{A, \text { even }}=m\left\{1, c_{t_{\alpha}}, c^{2}, c_{n}^{2}, c_{t_{\alpha}} c_{t_{\beta}}, c^{2} c_{t_{\alpha}}\right\}_{A}$. We do not discuss any details of the cumbersome derivation of the boundary conditions, but just give the final result, which is obtained after linearization in the evaporation velocity.

As an approximation to the Boltzmann equation, the R13 equations are expected to approximate, but not fully resolve, Knudsen layers [20]. To account for the related inaccuracies, in the interface conditions below we introduce ad-hoc corrections coefficients $\varpi_{\alpha}$ [21], which are expected to be of order unity. This approach is similar to what is usually done in jump and slip boundary conditions for hydrodynamics [9, 22].

The expression for evaporation flux results for $\hat{\Psi}_{A}=1$ as

$$
\rho V_{n}=\varpi_{V} \frac{\vartheta}{2-\vartheta} \sqrt{\frac{2}{\pi}}\left(\frac{p_{\text {sat }}\left(\theta_{L}\right)}{\sqrt{\theta_{L}}}-\frac{\Pi}{\sqrt{\theta}}\right),
$$

with the correction coefficient $\varpi_{V}$. Hence, the evaporation flux is determined through the difference between the saturation pressure $p_{\text {sat }}\left(\theta_{L}\right)$ of the liquid at the interface and the effective pressure $\Pi=\rho \theta+\frac{1}{2} \sigma_{n n}-\frac{1}{120} \frac{\Delta}{\theta}-\frac{1}{28} \frac{R_{n n}}{\theta}$. This expression is a generalization of the classical Hertz-Kundsen-Schrage law [23, 24, 25, 26] to the R13 equations. 
The other conditions are generalizations of the established wall boundary conditions for the R13 equations [14, 5], to which they reduce for non-evaporating interfaces $(\vartheta=0)$ :

Generalized slip condition:

$$
\sigma_{t_{\alpha} n}=-\varpi_{\sigma} \frac{\vartheta+\chi(1-\vartheta)}{2-\vartheta-\chi(1-\vartheta)} \sqrt{\frac{2}{\pi \theta}}\left(\Pi V_{t_{\alpha}}+\frac{1}{5} q_{t_{\alpha}}+\frac{1}{2} m_{t_{\alpha} n n}\right)-\rho V_{n} V_{t_{\alpha}}
$$

generalized temperature jump condition:

$$
q_{n}=-\varpi_{q} \frac{\vartheta+\chi(1-\vartheta)}{2-\vartheta-\chi(1-\vartheta)} \sqrt{\frac{2}{\pi \theta}}\left(2 \Pi\left(\theta-\theta_{L}\right)-\frac{\Pi}{2} V_{t}^{2}+\frac{1}{2} \theta \sigma_{n n}+\frac{\Delta}{15}+\frac{5}{28} R_{n n}\right)+\left[\frac{1}{2}\left(V_{t}^{2}-\theta_{L}\right)-\frac{5}{2}\left(\theta-\theta_{L}\right)\right] \rho V_{n}
$$

generalized interface conditions for higher moments:

$$
\begin{gathered}
m_{n n n}=\varpi_{m n} \frac{\vartheta+\chi(1-\vartheta)}{2-\vartheta-\chi(1-\vartheta)} \sqrt{\frac{2}{\pi \theta}}\left[\frac{2}{5} \Pi\left(\theta-\theta_{L}\right)-\frac{3}{5} \Pi V_{t}^{2}-\frac{7}{5} \theta \sigma_{n n}+\frac{\Delta}{75}-\frac{1}{14} R_{n n}\right]-\frac{2}{5}\left[\theta_{L}+\frac{3}{2} V_{t}^{2}\right] \rho V_{n} \\
m_{t_{\alpha} t_{\beta} n}=-\varpi_{m t} \frac{\vartheta+\chi(1-\vartheta)}{2-\vartheta-\chi(1-\vartheta)} \sqrt{\frac{2}{\pi \theta}}\left[\theta \sigma_{t_{\alpha} t_{\beta}}-\Pi V_{t_{\alpha}} V_{t_{\beta}}+\frac{R_{t_{\alpha} t_{\beta}}}{14}+\right. \\
\left.+\left(\frac{1}{5} \Pi\left(\theta-\theta_{L}\right)+\frac{1}{5} \Pi V_{t}^{2}-\frac{1}{5} \theta \sigma_{n n}+\frac{\Delta}{150}\right) \delta_{\alpha \beta}\right]+\rho V_{n}\left[V_{t_{\alpha}} V_{t_{\beta}}+\frac{1}{5}\left(\theta_{L}-V_{t}^{2}\right) \delta_{\alpha \beta}\right] \\
R_{t_{\alpha} n}=\varpi_{R} \frac{\vartheta+\chi(1-\vartheta)}{2-\vartheta-\chi(1-\vartheta)} \sqrt{\frac{2}{\pi \theta}}\left[\Pi \theta V_{t_{\alpha}}-\frac{11}{5} \theta q_{t_{\alpha}}-\frac{1}{2} \theta m_{t_{\alpha} n n}-\Pi V_{t_{\alpha}} V_{t_{\beta}} V_{t_{\beta}}+6 \Pi V_{t_{\alpha}}\left(\theta-\theta_{L}\right)\right]+ \\
+\left[7\left(\theta-\theta_{L}\right)+\theta_{L}-V_{t_{\beta}} V_{t_{\beta}}\right] \rho V_{t_{\alpha}} V_{n}
\end{gathered}
$$

The index $n$ refers to the interface normal, and indices $t_{\alpha}$ with $\alpha=1,2$ indicate the two tangential directions.

\section{Interface conditions for hydrodynamics}

In the hydrodynamic limit, only first order contributions in the Knudsen number are retained in the equations. For onedimensional processes, with transport only normal to the interface, and for only small deviations from equilibrium, the interface conditions are often written as $[27,16]$

$$
\left[\begin{array}{c}
\frac{p_{\mathrm{sat}}\left(\theta_{L}\right)-p}{\sqrt{2 \pi \theta_{L}}} \\
\frac{p}{\sqrt{2 \pi \theta_{L}}} \frac{\theta_{L}-\theta}{\theta_{L}}
\end{array}\right]=\left[\begin{array}{ll}
\hat{r}_{11} & \hat{r}_{12} \\
\hat{r}_{21} & \hat{r}_{22}
\end{array}\right]\left[\begin{array}{c}
{\left[\rho V_{n}\right]_{\mid \mathrm{NSF}}} \\
\frac{\left[q_{n}\right]_{\mathrm{NSF}}}{\theta_{L}}
\end{array}\right] \quad \text { with } \quad \hat{\mathbf{r}}_{\alpha \beta \mid \mathrm{corr}}=\left[\begin{array}{cc}
\frac{1}{\vartheta}-0.40044 & 0.126 \\
0.126 & 0.291
\end{array}\right]_{\alpha \beta},
$$

with a symmetric matrix $\hat{r}_{\alpha \beta}$ of Onsager coefficients, or resistivities [28]. The above values for $\hat{r}_{\alpha \beta \mid \text { corr }}$ are determined from exact calculations based on kinetic theory yield explicit corrections to account for Knudsen layer effects [19, 27], uncorrected values can be found in [16].

\section{1-D heat and mass transfer problems}

To put the R13 equations with evaporation/condensation to test, we now consider flows in simple one-dimensional geometry, and steady state. We ignore all details of mass and heat transfer through the liquid, and consider the temperature of the liquid at the interface as given. In particular, we consider systems where the normal of the interface points into the $x_{1}=x$ direction, and all flow properties are functions only of this coordinate. Moreover, we consider small deviations from an equilibrium rest state where vapor and liquid are at temperature $\theta_{0}$, hence the reference pressure is $p_{0}=p_{\text {sat }}\left(\theta_{0}\right)=\rho_{0} \theta_{0}$. We use the rest state data and the length scale $L$ to make the variables dimensionless. With all flows only in $x$-direction, the variable space is reduced to

$$
\hat{\rho}(\hat{x})=\frac{\rho(L \hat{x})}{\rho_{0}}, \hat{\theta}(\hat{x})=\frac{\theta(L \hat{x})}{\theta_{0}}, \hat{v}(\hat{x})=\frac{v_{x}(L \hat{x})}{\sqrt{\theta_{0}}}, \hat{\sigma}(\hat{x})=\frac{\sigma_{x x}(L \hat{x})}{p_{0}}, \hat{q}(\hat{x})=\frac{q_{x}(L \hat{x})}{p_{0} \sqrt{\theta_{0}}} .
$$


For convenience, the hats indicating dimensionless quantities will be omitted from now on.

The linearized dimensionless conservation laws (3) are easily integrated to give constant mass flow $J_{0}$, constant normal stress $P_{0}$ and constant energy flux $Q_{0}$.

$$
\rho v=J_{0}=\text { const }, \quad p+\sigma=P_{0}=\text { const } \quad, \quad \frac{5}{2} J_{0} \theta+q=Q_{0}=\text { const } .
$$

With this, the (linearized, dimensionless, one-dimensional) R13 constitutive equations for Maxwell molecules (10) reduce to

$$
\Delta=-12 \mathrm{Kn} \frac{\partial q}{\partial x}=30 J_{0} \mathrm{Kn} \frac{\partial \theta}{\partial x} \simeq 0, R=-\frac{16}{5} \mathrm{Kn} \frac{\partial q}{\partial x}=8 J_{0} \mathrm{Kn} \frac{\partial q}{\partial x} \simeq 0, \quad m=-\frac{4}{5} \mathrm{Kn} \frac{\partial \sigma}{\partial x} .
$$

The linearized balance equations for $x x$-component of stress (5) and $x$-component of heat flux (6) reduce to

$$
\frac{4}{5} \mathrm{Kn} \frac{\partial^{2} \sigma}{\partial x^{2}}=\frac{\sigma}{\mathrm{Kn}} \quad, \quad \frac{\partial \theta}{\partial x}=-\frac{4 \hat{Q}_{0}}{15 \mathrm{Kn}}-\frac{2}{5} \frac{\partial \sigma}{\partial x} .
$$

where $\hat{Q}_{0}=Q_{0}-\frac{5}{2} J_{0}$. These can be integrated easily to give

$$
\theta=K-\frac{4 \hat{Q}_{0} x}{15 \mathrm{Kn}}-\frac{2}{5} \sigma, \sigma=A \sinh \left[\sqrt{\frac{5}{4}} \frac{x}{\mathrm{Kn}}\right]+B \cosh \left[\sqrt{\frac{5}{4}} \frac{x}{\mathrm{Kn}}\right]
$$

where $K, A, B$ are constants of integration. We note that $\sigma$ is of Knudsen layer type, i.e., it decays exponentially away from the wall on the scale of the mean free path. We also note that classical hydrodynamics gives $\sigma=0$, and $\theta=K-\frac{4 \hat{Q}_{0} x}{15 \mathrm{Kn}}$, which is the case for $A=B=0$.

For the full solution, we have to find the six constants of integration $\left\{J_{0}, P_{0}, \hat{Q}_{0}, K, A, B\right\}$, from the appropriate boundary conditions for both sides. Equations $(15-20)$ reduce in linearized and dimensionless form to (no tangential components)

$$
\begin{aligned}
J_{0, n} & =\varpi_{V} \frac{\vartheta}{2-\vartheta} \sqrt{\frac{2}{\pi}}\left(p_{\text {sat }}\left(\theta_{L}\right)-P_{0}-\frac{1}{2}\left(\theta_{L}-\theta\right)+\frac{1}{2} \sigma\right), \\
\hat{Q}_{0, n} & =\varpi_{q} \frac{\vartheta+\chi(1-\vartheta)}{2-\vartheta-\chi(1-\vartheta)} \sqrt{\frac{2}{\pi}}\left(2\left(\theta_{L}-\theta\right)-\frac{1}{2} \sigma\right)-\frac{1}{2} J_{0, n}, \\
\frac{4}{5} \mathrm{Kn}\left[\frac{\partial \sigma}{\partial x}\right]_{n} & =\varpi_{m n} \frac{\vartheta+\chi(1-\vartheta)}{2-\vartheta-\chi(1-\vartheta)} \sqrt{\frac{2}{\pi}}\left[\frac{2}{5}\left(\theta_{L}-\theta\right)+\frac{7}{5} \sigma\right]+\frac{2}{5} J_{0, n} .
\end{aligned}
$$

Here it is assumed that all dimensionless pressures $p_{\text {sat }}\left(\theta_{L}\right), P_{0}$, and all dimensionless temperatures $\theta_{L}, \theta$, are close to unity. $J_{0, n}, \hat{Q}_{0, n}$ are the products of the flows $J_{0}, \hat{Q}_{0}$ with the normal at the respective boundary. For NSF, we consider the interface conditons (21).

\section{Half space problem}

In the classical problem of the steady evaporation from a planar infinite surface into a half-space [29, 30, 31], the evaporating liquid surface is kept at temperature $\theta_{L}$ with evaporation pressure $p_{s a t}\left(\theta_{L}\right)$. It is assumed that far downstream the flow is in a uniform equilibrium state characterized by pressure $p_{\infty} \leq p_{\text {sat }}$, temperature $\theta_{\infty}$ and bulk velocity $v_{\infty}>0$. Previous investigations [29] have shown that steady evaporation is possible only when the dowstream flow is subsonic and that only one of the three downstream flow parameters can be freely assigned. Therefore, the required solution is obtained by setting $Q_{0}=\sigma_{\infty}=0$ and hence the solution of the problem follows from (26) as:

$$
\theta(x)=\theta_{\infty}-\frac{2}{5} \sigma(x), \sigma(x)=A \exp \left[-\sqrt{\frac{5}{4}} \frac{x}{\mathrm{Kn}}\right] .
$$

With the evaporation speed $v_{\infty}$ prescribed, the downstream temperature $\theta_{\infty}$ and the pressure $p_{\infty}$ are fully determined by the relationships: 


$$
\alpha_{p}=\frac{p_{\text {sat }}\left(\theta_{L}^{0}\right)-p^{\infty}}{v_{\infty} / \sqrt{2}}, \quad \alpha_{\theta}=\frac{\theta_{L}-\theta_{\infty}}{v_{\infty} / \sqrt{2}} .
$$

The coefficients $\alpha_{p}$ and $\alpha_{\theta}$ can be determined by solving the linearized Boltzmann equation. For the Maxwell molecules potential considered here, an accurate approximate solution, for unit evaporation coefficient, has been obtained by Ytrehus by a moment method. Therefore, we compare results from R13 with Maxwell molecules and BGK model [32], Ytrehus' solution (Y) [29], and NSF, and will use the comparison to fit the corrections coefficients $\varpi_{\alpha}$. Since we only have two coefficients $\alpha_{p}, \alpha_{\theta}$, we have some freedom of choice, and, to keep things simple, we chose $\varpi_{m n}=1$, and fit only $\varpi_{V}, \varpi_{q}$. The various methods yield for the coefficients

$$
\begin{gathered}
\alpha_{p \mid \mathrm{R} 13}=\frac{\sqrt{\pi}}{4}\left[\frac{1}{2} \frac{1}{\varpi_{q}}+\frac{4}{\varpi_{V}}+\frac{\frac{2}{5}\left(1+\frac{1}{4 \varpi_{q}}\right)}{1+\frac{2}{15} \sqrt{10 \pi}}\right] \quad, \quad \alpha_{p \mid \mathrm{Y}}=\alpha_{p \mid \mathrm{NSF}}=2 \sqrt{\pi} \hat{r}_{11}=\frac{2}{\sqrt{\pi}}+\frac{9 \sqrt{\pi}}{16}=2.1254 \\
\alpha_{\theta \mid \mathrm{R} 13}=\frac{\sqrt{\pi}}{4}\left[\frac{1}{\omega_{q}}+\frac{\frac{4}{25}\left(1+\frac{1}{4} \frac{1}{\omega_{q}}\right)}{1+\frac{2}{15} \sqrt{10 \pi}}\right] \quad, \quad \alpha_{\theta \mid \mathrm{Y}}=\frac{\sqrt{\pi}}{4}=0.44311 \quad, \quad \alpha_{\theta \mid \mathrm{NSF}}=2 \sqrt{\pi} \hat{r}_{12}=0.44723
\end{gathered}
$$

For NSF the coefficients $\alpha_{p}$ and $\alpha_{\theta}$ are, apart from the factor $2 \sqrt{\pi}$, just the dimensionless resistivities $\hat{r}_{11}$ and $\hat{r}_{12}$ as defined in (21). In the R13 equations, we fit the free correction coefficients $\varpi_{V}, \varpi_{q}$, to the Ytrehus values for Maxwell molecules, to find $\varpi_{V}=0.9822, \varpi_{q}=1.126$. As expected, the correction coefficients are of order unity. Without the correction, i.e, for $\varpi_{V}=\varpi_{q}=1$, the R13 equations yield $\alpha_{p}=2.1208, \alpha_{\theta}=0.49383$, which is in good agreement for the pressure coefficient, but gives a $10 \%$ deviation for the temperature coefficient.

\section{Heat and mass transfer between two reservoirs}

For a closer look at the Knudsen layer, we consider heat and mass transfer between two liquid reservoirs at (dimensionless) locations $x= \pm \frac{1}{2}$, where the quantities at the two interfaces are indicated with superscripts 0,1 . The solution requires interface conditions on both sides of the domain. Note that at $x=\frac{1}{2}$ the interface normal points into the negative direction, so that, i.e., $J_{0, n}\left(x-\frac{1}{2}\right)=-J_{n, 0}\left(x+\frac{1}{2}\right)=J_{0}$ etc. We have three pairs of boundary conditions for $V_{n}, q_{n}, m_{n n n}$, given by (28), which are best applied by taking their pairwise sums and differences, respectively. After some calculation, the solution of the linear problem can be presented as

$$
P_{0}=\frac{p_{\text {sat }}\left(\theta_{L}^{0}\right)+p_{\text {sat }}\left(\theta_{L}^{1}\right)}{2}, \theta=\frac{\theta_{L}^{0}+\theta_{L}^{1}}{2}-\frac{4 \hat{Q}_{0}}{15 \mathrm{Kn}} x-\frac{2}{5} A \sinh \left[\sqrt{\frac{5}{4}} \frac{x}{\mathrm{Kn}}\right], \quad \sigma=A \sinh \left[\sqrt{\frac{5}{4}} \frac{x}{\mathrm{Kn}}\right] .
$$

Two of the pairwise sums of the boundary conditions give $0=\left(\theta_{L}^{0}-\theta^{0}\right)+\left(\theta_{L}^{1}-\theta^{1}\right)$, which was used in the above.

The evaporation fluxes $J_{0}$, the heat flux $Q_{0}$ and the amplitude of the Knudsen layer $A$ are obtained from a linear system which results from subtracting the interface conditions at both sides:

$$
\begin{aligned}
J_{0} & =\varpi_{V} \frac{\vartheta}{2-\vartheta} \sqrt{\frac{2}{\pi}} \frac{1}{2}\left(p_{\text {sat }}\left(\theta_{L}^{0}\right)-p_{\text {sat }}\left(\theta_{L}^{1}\right)+\frac{2 Q_{0}}{15 \mathrm{Kn}}+\frac{\theta_{L}^{1}-\theta_{L}^{0}}{2}-\frac{3}{5} A \sinh \left[\sqrt{\frac{5}{4}} \frac{1}{2 \mathrm{Kn}}\right]\right), \\
\hat{Q}_{0} & =-\varpi_{q} \frac{\vartheta+\chi(1-\vartheta)}{2-\vartheta-\chi(1-\vartheta)} \sqrt{\frac{2}{\pi}}\left(\frac{4 Q_{0}}{15 \mathrm{Kn}}+\theta_{L}^{1}-\theta_{L}^{0}+\frac{3}{10} A \sinh \left[\sqrt{\frac{5}{4}} \frac{1}{2 \mathrm{Kn}}\right]\right)-\frac{J_{0}}{2}, \\
A & =-\varpi_{m n} \frac{\frac{\vartheta+\chi(1-\vartheta)}{2-\vartheta-\chi(1-\vartheta)}}{2 \sqrt{5} \cosh \left[\sqrt{\frac{5}{4}} \frac{1}{2 \mathrm{Kn}}\right]} \sqrt{\frac{2}{\pi}}\left(\frac{4 Q_{0}}{15 \mathrm{Kn}}+\theta_{L}^{1}-\theta_{L}^{0}+\frac{39}{5} A \sinh \left[\sqrt{\frac{5}{4}} \frac{1}{2 \mathrm{Kn}}\right]\right)+2 J_{0} .
\end{aligned}
$$

Solving the system, and inserting the results into (32) yields detailed profiles of temperature and stress. The NSF solution results by replacing $(34)_{3}$ with $A=0$. We compare R13 and NSF predictions to DSMC simulations (all with 

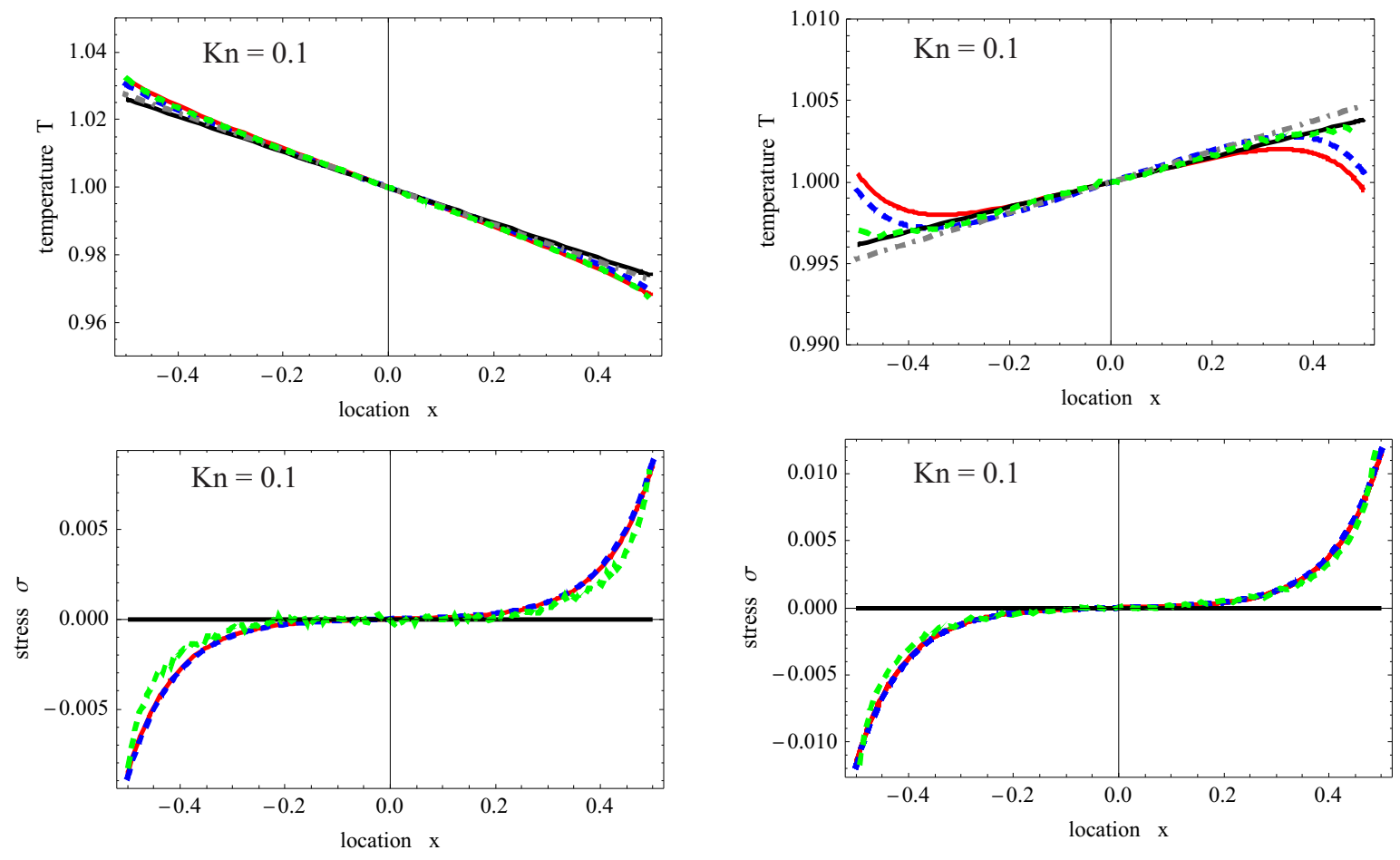

normal temperature profile

inverted temperature profile

FIGURE 1. Normal (left) and inverted (right) temperature profile: Temperature and stress profiles for Kn=0.1. Comparison of DSMC (green, dashed), R13 with corrected BC (red, continuous), R13 with uncorrected BC (blue, dashed), NSF with Knudsen layers correction (black, continuous), NSF without correction (grey).

$\chi=1$ ) for cases with normal temperature profile, and inverted temperature profile. Dimensionless temperatures and saturation pressure of the two liquids are prescribed as $\theta_{L}^{0,1}=1 \pm \Delta \theta, p_{\text {sat }}\left(\theta_{L}^{0,1}\right)=1 \pm \Delta p$, with $\Delta p=0.075$, and $\Delta \theta=0.05$ for the normal temperature profile, $\Delta \theta=0.1$ for the inverted profile.

Figure 1 shows on the left temperature and stress profiles for the case of normal temperature profile, which has the temperature gradient pointing from hot (left) to cold (right), comparing DSMC with R13 and NSF solutions for $\mathrm{Kn}=0.1$. The figures indicates good agreement of R13 results with DSMC for temperature and stress profiles, with somewhat better agreement for the boundary conditions with adjusted correction coefficients $\varpi_{\alpha}$ (red curve) over the uncorrected coefficients $\left(\varpi_{\alpha}=1\right.$, blue). NSF with (black) and without (grey) Knudsen layer correction cannot match the temperature curve, and give $\sigma=0$, while R13 can match the stress quite well. The upper block in Table 1 gives the corresponding values of mass and heat flux, and it becomes clear that all theories give the mass flux $J_{0}$ in good agreement to DSMC, while the heat flux $\hat{Q}_{0}$ exhibits considerable deviations.

The plots on the right of Figure 1, and the lower block of Table 1 show results for the inverted temperature profile, which has the temperature gradient pointing from cold (right) to hot (left), again comparing DSMC with R13 and NSF solutions for $\mathrm{Kn}=0.1$. In this case, the R13 equations with (red) and without (blue) correction overemphasize the

TABLE 1. Mass and heat flux, and relative error to DSMC in \%, for vapor enclosed between two liquid surfaces at $\mathrm{Kn}=0.1$, determined from DSMC, R13, and NSF.

\begin{tabular}{|l|l||l||l|l||l||l||l||l|l|l|l|}
\hline & & DSMC & R13, corr & error & R13 & error & NSF, corr & error & NSF & error \\
\hline normal & $J_{0}$ & $\mathbf{0 . 0 2 9 4}$ & 0.0289 & 1.7 & 0.0287 & 0.7 & 0.0303 & 3.1 & 0.0310 & 5.4 \\
& $\hat{Q}_{0}$ & $\mathbf{0 . 0 1 7 2}$ & 0.0215 & 25 & 0.0203 & 18 & 0.0196 & 14 & 0.0206 & 20 \\
\hline \multirow{2}{*}{ inverted } & $J_{0}$ & $\mathbf{0 . 0 5 0 3}$ & 0.0506 & 0.6 & 0.0510 & 1.4 & 0.0504 & 0.2 & 0.0540 & 7.4 \\
& $\hat{Q}_{0}$ & $\mathbf{- 0 . 0 0 2 7 4}$ & -0.00307 & 12 & -0.00395 & 44 & -0.00288 & 5.1 & -0.00353 & 29 \\
\hline
\end{tabular}


Knudsen layer for the temperature, while NSF with correction (black) gives an excellent match. While NSF gives $\sigma=0$, the R13 prediction for stress agress well with DSMC. All theories give the mass flux $J_{0}$ in good agreement to DSMC, while the heat flux $\hat{Q}_{0}$ exhibits considerable deviations. Interestingly, NSF with corrected boundary conditions gives the best results, while NSF with uncorrected boundary conditions deviates most. This points to the importance of adjusting the boundary conditions properly.

\section{Conclusions}

The evaluation of evaporation/condensation processes with the R13 and NSF equations based on non-equilibrium boundary conditions shows that both can produce results in reasonable agreement with DSMC calculations. R13 can, in principle, resolve more detail, including Knudsen layers for the temperature, and-in particular-for normal stress, which is not accessible for NSF. It should be noted that the NSF boundary conditions used are carefully corrected based on the 2nd law of thermodynamics and fitting to exact solutions, which give the Onsager coefficients in the matrix $\hat{r}_{\alpha \beta}$. A similar fitting procedure will be required for the R13 equations, which here were corrected only in a rather ad-hoc way, by means of the coefficents $\varpi_{\alpha}$. A more careful study of the boundary conditions based on a full 2nd law analysis with proper Onsager coefficients [20] should lead to improved agreement between R13 and DSMC, or other exact solutions of the Boltzmann equation.

\section{Acknowledgement}

Support from the Natural Sciences and Engineering Research Council (NSERC) is gratefully acknowledged.

\section{REFERENCES}

[1] H. Struchtrup and M. Torrilhon, Phys. Fluids 15(9), 2668-2680 (2003)

[2] M. Torrilhon and H. Struchtrup, J. Fluid Mech. 513, 171-198 (2004)

[3] H. Struchtrup, Macroscopic Transport Equations for Rarefied Gas Flows, Springer, Heidelberg 2005

[4] H. Struchtrup and P. Taheri, IMA J. Appl. Math. 76(5), 672-697 (2011)

[5] M. Torrilhon, Ann. Rev. Fluid Mechanics 48, 429-458 (2016)

[6] H. Struchtrup, Multiscale Model. Simul. 3(1), 211-243 (2004)

[7] H. Struchtrup, Phys. Fluids 16(11), 3921-3934 (2004)

[8] S. Chapman, T.G. Cowling, The Mathematical Theory of Non-Uniform Gases. Cambridge Univ. Press 1970

[9] C. Cercignani, Theory and Application of the Boltzmann Equation. Scottish Academic Press, Edinburgh 1975

[10] D. Burnett, Proc. Lond. Math. Soc. 40, 382-435 (1936)

[11] M.Sh. Shavaliyev, J. Appl. Maths. Mechs. 57(3), 573-576 (1993)

[12] A.V. Bobylev, Sov. Phys. Dokl. 27, 29-31 (1982)

[13] H. Grad, Principles of the Kinetic Theory of Gases, in Handbuch der Physik XII, Springer, Berlin 1958

[14] M. Torrilhon and H. Struchtrup, J. Comp. Phys. 227, 1982-2011 (2008)

[15] J.C. Maxwell, Phil. Trans. Roy. Soc. 170, 231-256 (1879)

[16] J.P. Caputa and H. Struchtrup, Physica A 390, 31-42 (2011)

[17] A.S. Rana, M. Torrilhon, and H. Struchtrup, J. Comp. Phys 236, 169-186 (2013)

[18] I. Kuscer and M. Robnik, J. Phys. A: Math. Gen. 13, 621 (1980)

[19] J.W. Cipolla, H. Lang, and S.K. Loyalka, J. Chem. Phys. 61, 69-78 (1974)

[20] A.S. Rana and H. Struchtrup, Phys. Fluids 28, 027105 (2016)

[21] M. Torrilhon and H. Struchtrup, ASME J. Heat Transfer 131, 033103 (2009)

[22] H. Struchtrup, Physica A 387, 1750-1766 (2008)

[23] H. Hertz, Annalen der Physik 253, 177-193 (1882).

[24] M. Knudsen, Annalen der Physik 352, 697-708 (1915).

[25] R.W. Schrage, A Theoretical Study of Interphase Mass Transfer. Columbia University Press, New York 1953

[26] M. Bond and H. Struchtrup, Phys. Rev. E 70, 061605 (2004)

[27] S. Kjelstrup, D. Bedeaux, Non-Eq. Thermodynamics of Heterogeneous Systems, World Scientific, 2008.

[28] S.R. de Groot and P. Mazur, Non-Equilibrium Thermodynamics, Dover, 1984

[29] T. Ytrehus, Kinetic Theory Description and Experimental Results for Vapor Motion in Arbitrary Strong Evaporation. Technical Note 112, von Karman Institute for Fluid Dynamics, 1975

[30] Y. Sone, TTSP 29, 227-260 (2000)

[31] A. Frezzotti, Europ. J. Mechanics-B/Fluids 26, 93-104 (2007)

[32] Y.P. Pao, Phys. Fluids 14, 306-312 (1971), Phys. Fluids 14, 1340-1346 (1971) 